\title{
Un projet pour l'éducation plurilingue et interculturelle
}

Marc-André Jullian

\section{(2) OpenEdition \\ Journals}

Édition électronique

URL : http://journals.openedition.org/esp/2713

DOI : 10.4000/esp. 2713

ISSN : 2532-0319

Éditeur

Centre d'Information sur l'Éducation Bilingue et Plurilingue

Édition imprimée

Date de publication : 1 décembre 2013

Pagination : $3-13$

ISSN : 1127-266X

\section{Référence électronique}

Marc-André Jullian, « Un projet pour l'éducation plurilingue et interculturelle », Éducation et sociétés plurilingues [En ligne], 35 | 2013, mis en ligne le 01 février 2020, consulté le 17 février 2021. URL http://journals.openedition.org/esp/2713 ; DOl : https://doi.org/10.4000/esp.2713 


\section{UN PROJET POUR L'ÉDUCATION PLURILINGUE ET INTERCULTURELLE}

\section{Marc-André JULLIAN}

Questo testo è la presentazione di uno strumento pedagogico creato nel corso e per mezzo di un progetto europeo franco-valdostano condotto principalmente nelle lingue regionali insegnate nelle due regioni. L'occitano e il catalano, il franco-provenzale, il titsch e il töitschu cercheranno di mostrare nell'universo delle altre lingue e all'interno di questo progetto il posto che occupano e la loro piena legittimità nel contesto dello sviluppo o dell'educazione plurilingue ed interculturale.

Aquel texte se vòl èsser la presentacion del projècte que menèron las classas bilingüas pendent doas annadas sul camin de l'educacion plurilingüe e interculturala a l'entorn dels contes e legendas dins las lengas regionalas de l'Acadèmia de Montpelhièr e la Val d'Aosta. Lo punt fort n'es la produccion d'àlboms inventats e illustrats per d'escolans de l'escòla mairala a la fin del primari e d'una classa de collègi, integrats dins de sacas d'istòrias.

This text presents a pedagogical tool created through and during a European France-Aosta Valley project mainly based on the regional languages taught in the two parts of the country. Occitan and catalan, francoprovençal, titsch and toitschu demonstrate the place they occupy in the universe of the other languages and their legitimate nature in the service of plurilingual and intercultural teaching and education.

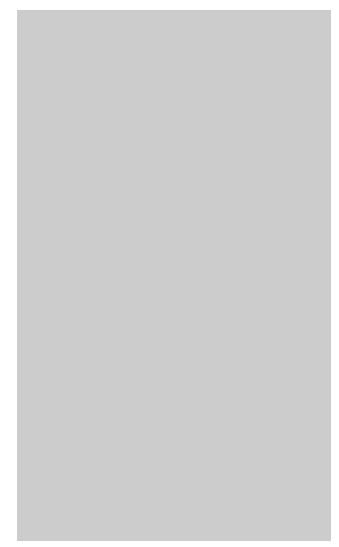

Tnverser l'ordre des mots pour faire sien un titre d'article est chose facile, mais je ne peux faire autrement qu'évoquer ici, en préambuLe, l'origine d'un projet qui a impliqué plus de 25 partenaires attachés à l'Éducation durant plus de 2 ans.

Le titre original auquel je fais référence est "L'éducation plurilingue et interculturelle comme projet" produit par Marisa Cavalli, Daniel Coste, Alexandru Crisan et Piet-Hein van de Ven en 2009 pour la plateforme de ressources et de références pour l'éducation plurilingue et interculturelle au sein de la commission des politiques linguistiques du Conseil de l'Europe. Cette étude détaillée qui donne de multiples définitions attachées aux notions de plurilinguisme et d'interculturalité tombait à point nommé dans une phase de recherche pour redynamiser l'enseignement bilingue français/langue régionale que j'avais pour mission de développer dans l'exercice de mes fonctions. Ce texte met en valeur plu- 
Un projet pour

l'éducation plurilingue

et interculturelle

M.-A. JULLIAN

\section{LA PROBLÉMATIQUE DE L'ENSEIGNEMENT BILINGUE FRANÇAIS/OGGITAN:}

D'UN PROJET

À L'AUTRE sieurs points dont: la notion de projet qui vient étayer une entreprise d'avenir pour l'enseignement des langues, la formation des enfants et des enseignants. Je n'en citerai ici qu'un seul passage qui me semble résumer, et entamer à la fois, une démarche à suivre contre les représentations sociales et parfois institutionnelles, pour tenter de convaincre par des résultats. Il s'agit des "fonctions multiples des langues 'autres' dans l'éducation plurilingue et interculturelle", considérant que:

- $\quad$ "toutes les langues ont une valeur indépendamment de leur statut dans une société donnée (langues officielles, régionales, minoritaires, de la migration,...) et de leurs statuts didactiques (langue 1, langue 2, langues "étrangères" d'origine, langues étrangères vivantes, langues classiques)

- les diverses langues qui font partie du répertoire personnel des apprenants, mais qui n'entrent pas parmi les langues enseignées comme matières du programme, revêtent une importance éducative toute particulière: ce sont des langues que l'École à travers des approches plurielles et partielles, peut valoriser, en confortant l'identité des apprenants et en leur donnant d'égales opportunités de réussite scolaire".

La prise en compte et la mise en valeur des langues et des cultures, sans exclusive, nous conduira de l'enseignement bilingue vers un développement du plurilinguisme comme projet pédagogique et didactique.

Comme dans la plupart des régions françaises possédant une langue régionale reconnue, l'enseignement bilingue français/occitan inscrit dans l'enseignement public souffre des représentations plutôt négatives ou tout au moins de l'ignorance convergente dont font preuve trop souvent les acteurs de l'éducation (parents, enseignants, responsables administratifs). S'ajoute à cela une confusion entre deux organisations de l'éducation aux langues étrangères entre les sections bilangues et celle de section bilingue; la première autorisant l'enseignement de deux langues autres que le français (enseignées comme matière), la seconde incluant l'apprentissage de la langue 2 (dont le français - langue de scolarisation) dans l'apprentissage des disciplines.

De cette situation plutôt néfaste pour l'avenir des cursus bilingues, il devenait nécessaire de construire un projet porteur d'ouverture qui tenterait de promouvoir la langue régionale et l'enseignement bi-plurilingue dans l'intérêt des enfants déjà inscrits ou en train de l'être dans les sites de l'académie de Montpellier.

Les lectures ciblées de nombre de linguistes et spécialistes de la question de l'apprentissage des langues (1), les rencontres lors de colloques, formations spécifiques, ont largement servi la nécessité d'aller se rendre compte in situ du fonctionnement du bi- plurilinguisme en Vallée d'Aoste comme souvent décrit dans les expérimentations didactiques et 
Un projet pour

l'éducation plurilingue

et interculturelle

M.-A. JULLIAN

\section{QUELQUES}

ELLÉMENTS

SOGIOLINGUISTI-

QUES ET

ECONOMIQUES pédagogiques.

D'une première rencontre à laquelle ont participé la plupart des formateurs en langues régionales de l'académie de Montpellier (occitan et catalan), est née une volonté de poursuivre les échanges et d'associer les enseignants chargés de classes bilingues, de part et d'autre de la frontière. Une base de réflexion a été lancée autour des contes et légendes via la plateforme e.Twinning et ces échanges ont permis de fédérer une grande majorité des maitres et maitresses languedociens et valdôtains sur un besoin de formation et d'échanges pédagogiques. La connaissance réciproque des fonctionnements scolaires devenait quant à elle indispensable, tout comme le développement de l'utilisation des techniques de communication (TICE). La concertation et les relations professionnelles entre les formateurs de l'Assessorat de l'Éducation et de la Culture (Mme Vernetto en particulier) et de l'académie de Montpellier ont grandement favorisé la mise en place d'une aventure beaucoup plus ambitieuse, impliquant deux collectivités territoriales comme porteurs officiels d'un projet européen Comenius Regio.

"La Vallée d'Aoste est l'une des cinq régions italiennes bénéficiant d'un statut d'autonomie (Loi constitutionnelle n 4 du 26 février 1948) qui reconnait deux langues officielles (français et italien) enseignées pour un même nombre d'heures dans tous les établissements scolaires de la région, ainsi qu'une troisième langue, l'allemand, pour les communes Walser de la Vallée du Lys. Entre les aspects les plus caractéristiques de la Vallée d'Aoste, sa configuration linguistique joue un des premiers rôles. À cette configuration contribuent naturellement les présupposés bilingues statutaires mais aussi une constellation plurilingue bien enracinée et diffusée inhérente au domaine des langues d'utilisation lors de la communication quotidienne, qui regroupe les variétés linguistiques natives de la zone (le francoprovençal de la Vallée d'Aoste), ainsi que les variétés minoritaires d'importation plus ancienne (telles que les dialectes alémaniques des Walser de la Vallée du Lys ou le dialecte piémontais parlé dans la Basse Vallée) ou plus récente (les différents dialectes italiens et le langues de l'immigration).

Suite aux lois nationale (482/99) et régionale (18/2005) pour la promotion des langues minoritaires et régionales, l'Assessorat de l'éducation et de la culture de la Vallée d'Aoste a mis en œuvre une série d'initiatives diversifiées en faveur de ces langues: formation des enseignants, publication d'outils et de ressources pédagogiques, ateliers de sensibilisation à l'intention des élèves de tous les niveaux scolaires. Ces initiatives font partie intégrante des actions envisagées pour l'éducation plurilingue et la promotion de la diversité linguistique et culturelle (dimension européenne de l'éducation), dans le cadre d'une approche holistique de l'enseignement/apprentissage des langues qui intègre la valorisation des langues régionales, au lieu de proposer une approche centrée exclusi- 
Un projet pour l'éducation plurilingue et interculturelle

M.-A. JULLIAN

\section{DES OBJECTIFS CIBLÉS}

vement sur les langues minoritaires, ce qui constituerait une espèce de ghettoïsation de ces langues et de folklorisation des cultures qu'elles véhiculent" (Gabriella Vernetto in dossier de candidature au projet Comenius Regio 2011).

L'académie de Montpellier, quant à elle, correspond à la région Languedoc Roussillon, composée d'une large bande littorale méditerranéenne à densité de population importante et un arrière pays allant jusqu'aux plateaux auvergnats où la population est plus clairsemée et moins diversifiée à l'exception de quelques villes plus importantes (Nîmes,, Alès, Lodève, Carcassonne...). Elle a la particularité d'avoir deux langues régionales sur son territoire: l'occitan et le catalan. Langues de même origine, leur statut linguistique a évolué différemment de par une influence économique/politique de la Catalogne sud (Espagne) très importante pour le catalan. L'occitan est langue passerelle entre les régions occitanophones de l'Espagne et des vallées piémontaises italiennes. Elles ont toutes deux le même statut linguistique dans le domaine de l'éducation nationale, au titre des langues régionales, et sont enseignées sous plusieurs formes, dont l'enseignement bilingue à parité horaire (50\% du temps en français et $50 \%$ du temps en langue régionale) dans l'enseignement public (sept sites au total pour l'occitan et quinze pour le catalan).

À l'issue de l'évaluation des besoins, deux catégories d'objectifs ont constitué la base de l'architecture de notre projet:

- les objectifs centraux: le développement des programmes éducatifs par projets pour promouvoir la transversalité des curricula et des compétences de base;

- la promotion de la dimension européenne et internationale des établissements scolaires;

- le soutien au bi-plurilinguisme et au dialogue interculturel;

- l'emploi des TICE et du multimédia en tant qu'outils de partage et de collaboration.

et les objectifs dits "concrets" du partenariat: favoriser la dimension européenne à travers une communication de pratiques pédagogiques, par la mise en place d'une sorte de laboratoire bilatéral "permanent" de réflexion et d'essais en pédagogie du biplurilinguisme avec des enfants de 3 à 11 ans qui se rejoignent de façon pertinente dans les objectifs du programme Comenius:

- Améliorer la qualité des mobilités élèves ainsi que des personnels enseignants et non enseignants, en augmenter le nombre dans tous les États membres.

- Améliorer la qualité des partenariats entre établissements de l'enseignement scolaire des États membres, en accroitre le nombre avec pour but d'impliquer au moins 3 millions d'élèves dans des activités éducatives 
Un projet pour

l'éducation plurilingue

et interculturelle

M.-A. JULLIAN

DES PARTENAIRES CHOISIS

DES ACTIONS SPÉCIFIQUES conjointes pendant la durée de la programmation

- Encourager l'apprentissage des langues vivantes étrangères

- Soutenir le développement de contenus, services, pédagogies et pratiques qui s'appuient sur l'utilisation des TICE dans l'éducation et la formation tout au long de la vie

- Améliorer la qualité et la dimension européenne de la formation des enseignants

- Soutenir les améliorations en matière d'approches pédagogiques et de gestion des établissements de l'enseignement scolaire

La structure particulière du Comenius Regio implique des collectivités territoriales, des établissements scolaires et des acteurs de l'éducation informelle. Vingt-sept structures se sont donc regroupées pour le bon déroulement du projet: 2 collectivités territoriales que sont l'Assessorat de l'Éducation à Aoste et la ville du Vigan, commune rurale cévenole de 4000 habitants, 15 établissements ou institutions scolaires, 6 organismes attachés à l'éducation par leurs activités de recherche, de promotion linguistique ou de diffusion d'ouvrages littéraires et 4 structures expertes de tutelle des enseignants et formateurs français. Un ensemble à coordonner et informer régulièrement pour que le projet suive les perspectives et actions définies autour des objectifs retenus.

Deux volets d'actions conjuguées ont mobilisé les acteurs du projet autour de la formation didactique des enseignants et la création pédagogique par les élèves. Cette vision globale de la formation au bi-plurilinguisme et la mise en pratique en classe a permis la production d'un outil de développement des pratiques plurilingues, conforme aux préconisations de Michel Candelier (Université du Maine) et Luisa Revelli (Université d'Aoste) qui nous ont présenté leurs réflexions sur le plurilinguisme et l'enseignement précoce des langues ou encore la didactique intégrée des langues.

Née en 1997 en Angleterre, l'initiative "sac d'histoires" a évolué au Québec où elle a pris une dimension bilingue. C'est en Suisse (2007) où le concept est devenu porteur de plurilinguisme et s'est doté de multiples atouts pour motiver sa circulation dans les familles grace aux travaux de Christiane Perregaux et Élisabeth Zurbriggen (coordinatrice pédagogique SCOP-DEP- Genève) et qui a fait l'objet de plusieurs publications. Nous sommes donc les premiers à les diffuser en Italie et en France avec une adaptation supplémentaire.

Le statut particulier des langues régionales, reconnues par l'administration française mais pas encore entièrement considérées comme support d'apprentissage des langues, a induit une démarche intégrative, associant le fait culturel à l'activité linguistique. Après la présentation et 
Un projet pour

l'éducation plurilingue

et interculturelle

M.-A. JULLIAN

UNE MÉMOIRE VIRTUELLE l'étude de contes et légendes locales ou régionales, de productions thématiques en classe, la plupart des productions est fondée sur un élément culturel fortement attaché à la région d'implantation de l'école, écrit ou transcrit dans la langue régionale d'enseignement: occitan, catalan, francoprovençal, titsch ou toïsschu.

D'une péniche voulant des couleurs sur le canal du Midi à la naissance du glacier du Ruitor (2), chaque conte a été écrit, corrigé, repris, relu, validé puis illustré en classe. L'aide d'un comité scientifique composé de personnalités de l'éducation ou de la recherche en linguistique a permis une production finale conforme aux attentes et aux normes linguistiques espérées (3).

Dans l'optique des sacs d'histoires et du développement du plurilinguisme par la valorisation des langues de l'environnement, chaque conte a été traduit dans plusieurs langues, en fonction des communautés représentées au moment de l'enquête auprès des familles, puis édité en version bilingue dont le français comme langue du projet. Les langues régionales déjà citées sont, de fait, au même niveau que les langues de large diffusion (anglais, allemand, espagnol, italien) et que les langues moins répandues ou de l'immigration (portugais, turc, japonais, albanais, arabe). La richesse de notre projet réside également dans sa particularité d'ouverture à d'autres langues, non encore présentes sur les territoires concernés. En effet, une version de chaque album dite à "pochettes" permettra d'enrichir l'inventaire des traductions. Parallèlement aux albums, chaque sac est composé, comme dans les productions suisses, d'un CD audio autorisant l'écoute de toutes les versions linguistiques enregistrées, d'un glossaire permettant un réel retour des familles et une étude des langues en approches plurielles (Candelier; Revelli; Zurbriggen; Dominguez-Fonseca: interventions en formation), un jeu lié à l'histoire donnant à l'enfant une place centrale lors de la découverte du sac en famille, et enfin une surprise comme élément de motivation pour l'ensemble de ces activités.

La richesse des productions et la qualité des interventions, la multitude de documents édités ou les nouvelles du projet ne pouvaient être mises de côté au risque de les perdre. Un site spécifique pour tous ces éléments a été spécialement créé par les experts en nouvelles technologies de l'information de l'assessorat de l'éducation valdôtain. http://www.scuole.vda.it/comenius/ Composé d'un double volet, il répond au premier objectif d'information sur l'ensemble du projet, de son organisation et déroulement, de ses nouvelles. Le second volet est d'ordre plus réservé et constitue la banque de données à disposition des utilisateurs des sacs d'histoires tant du point de vue didactique que comme matériel pédagogique. Il continuera à vivre en fonction des ini- 


Un projet pour
I'éducation plurilingue
et interculturelle
M.-A. JuLLIAN
UN PROJET OUVERT:
DES LANGUES
RÉGIONALES
AUX LANGUES
ÉTRANGĖRES DE
PROXIMITÉ OU DE
L'IMMIGRATION.

D'UNE PHASE DE CONCEPTION/ CONSTRUCTION VERS UNE PHASE D'UTILISATIONÉVALUATION tiatives et de la poursuite de l'opération "sacs d'histoires".

Comment aborder les cultures et les partager pour accéder au "dialogue interculturel" comme précisé dans nos objectifs? Notre réflexion a pris très rapidement en compte un élément commun à toutes les cultures: le conte ou la légende, domaine transversal et universel à tous les peuples et à toutes les langues. Choisir d'intégrer la culture régionale dans les productions linguistiques n'est donc pas un hasard mais un vecteur d'ouverture à la connaissance de tous les autres et bien sûr d'échanges futurs par comparaison tant en ressemblances qu'en différences. La circulation des sacs d'histoires dans les familles offre une double plus-value pédagogique: d'une part ils mettent en valeur toutes les langues de l'environnement des élèves, y compris les langues de l'immigration, avec toutes les représentations qui s'y rattachent tant du point de vue des locuteurs que de celui de la société; de l'autre ils impliquent les familles dans un va-et-vient entre l'environnement familial et l'école. Chaque enfant partant normalement à la maison avec le sac contenant la version bilingue du conte correspondant à sa langue d'origine, voilà l'occasion de mettre en contact les deux univers des langues et des cultures. Il est probable que l'existence de la même légende ou de la thématique très proche suscite un échange intergénérationnel et la transmission de savoirs. C'est alors à l'école qu'il conviendra d'exploiter cette ouverture aux cultures. Plusieurs axes sont envisageables tels les arbres à histoires rassemblant les titres de livres lus ou racontés à la maison, l'utilisation des glossaires (Zurbriggen 2011, Conférence d'Élisabeth Zurbriggen lors du séminaire d'ouverture Aoste 10-2011).

À l'heure actuelle, nos collections de sacs sont terminées et sont parties dans les sites partenaires du projet. C'est ce que nous appelions la phase de réalisation. Et si l'idée semble tout à fait intéressante, sa conception/construction n'a pas été sans poser quelques problèmes. Le choix des premiers contes ou légendes, la détermination du thème central de la production, l'attachement culturel, et ensuite l'imagination des auxiliaires d'accompagnement constituant le "concept" ont suscité nombre de questionnements et d'échanges au cours des deux années d'élaboration. Il nous revient maintenant la deuxième phase, celle de l'utilisation et de l'évaluation de notre opération. Nous basons nos premières réflexions sur les expériences déjà menées à Genève et diffusées par vidéofilm interposé. Les commentaires et réflexions des parents interrogés sont porteurs d'espérances. Qu'en sera-t-il de notre côté? Les premières présentations aux parents ou enseignants sont prometteuses, car plusieurs établissements scolaires valdôtains se sont inscrits dès ce mois de septembre pour l'utilisa- 
Un projet pour

l'éducation plurilingue

et interculturelle

M.-A. JULLIAN

LE PROJET COMME VECTEUR DE MODIFICATIONS DES PRATIQUES tion et l'expérimentation de nos sacs d'histoires. Il est certain que tout sera mis en œuvre pour un suivi et une évaluation des retombées pédagogiques dans le domaine des transmissions linguistiques et culturelles et des pratiques plurilingues.

La spécificité des albums à format "pochette" permettra une ouverture supplémentaire par prolongement dans la création. La modification de la carte sociologique des langues représentées dans un territoire pourra donc être prise en compte par la traduction et l'enregistrement du ou de certains contes dans les langues non encore présentes en édition ou disponibles sur le site dédié.

La deuxième partie de l'évaluation portera également de façon interne sur l'image des langues, et en particulier des langues "modimes" c'est-à-dire moins dites et moins enseignées. Il s'agira de vérifier si pour la plupart, elles demeurent dans leurs représentations sociales quelque peu stéréotypées et ensuite de tenter d'agir pour les modifier. Ces langues souvent considérées comme non "capitalisables", c'est-à-dire qui ne serviraient pas dans le monde d'aujourd'hui à un quelconque rapport économique, sont comme toutes les autres, porteuses d'une culture et d'une autre façon de voir le monde. Au cœur des activités d'exploitation pédagogiques, l'occitan au même titre qu'une autre langue romane ne servira-t-il pas à repérer les ressemblances, à fédérer les familles de mots, à construire un savoir plurilingue utile dans l'apprentissage général des langues? De même pour les langues d'origine arabe d'où sont issus bien des emprunts en français par exemple et qui sont riches d'une vision scientifique (liaison entre chiffre et zéro par exemple).

Au cours de ces deux années de suivi du déroulement du projet, certaines évaluations ont eu lieu, et notamment quant aux modifications des pratiques pédagogiques et des rapports au travail en équipe. De façons très différentes, certains enseignants ont partagé le travail à accomplir, en fonction de leurs classes (âge des élèves, compétences, temps disponible) ou de la configuration organisationnelle et géographique de l'établissement/institution. Il est à relever certaines remarques très positives quant à l'ouverture autour du projet. Plusieurs échanges donc, entre les enseignants d'une même école, d'écoles de niveaux d'enseignement différents (maternelle/élémentaire/collège)(4) ont eu lieu pour l'organisation du projet, les répartitions de travaux, les décisions et validations; entre les classes où les élèves ont été répartis en groupes ou ateliers pour les réflexions, préparations, confrontations; pour des présentations (lectures d'ébauches de textes, illustrations, mise en scène...) 


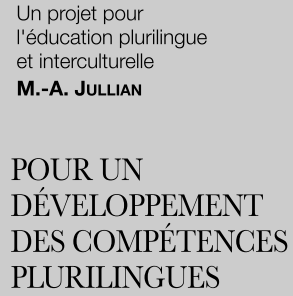

POUR UNE

VALEUR AJOUTÉE EUROPÉENNE
D'autres situations n'ont pas permis ce travail de collaboration interne et locale et l'échange s'est réalisé uniquement à travers les modules de formation. La deuxième phase d'exploitation des sacs devrait permettre une poursuite de ce processus de communication pour gérer la circulation, l'évaluation et l'enrichissement des productions.

Les objectifs du projet sont liés aux approches plurielles dans l'apprentissage des langues, en suivant les conseils de Michel Candelier qui préconise l'utilisation de plusieurs langues pour acquérir des notions par un effet de retour et d'éclairage sur la L2 pour mieux comprendre la L1. Cette didactique des langues intégrée permet de jouer sur les particularités de chacune pour en comprendre le fonctionnement ou au contraire sur leur proximité pour favoriser l'intercompréhension, quand il s'agit de langues parentes. Et c'est la multiplication de ces opérations qui doit contribuer à la formation des compétences plurilingues mais également interculturelles comme définies ci après: On désignera par compétence plurilingue et pluriculturelle, la compétence à communiquer langagièrement et à interagir culturellement possédée par un locuteur qui maîtrise, à des degrés divers, plusieurs langues et a, à des degrés divers, l'expérience de plusieurs cultures, tout en étant à même de gérer l'ensemble de ce capital langagier et culturel... (Coste, Moore et Zarate,2009,p.11) tout en prenant garde de valoriser la variation intra et interlinguistique autant que la différenciation intra et interculturelle pour "aider les apprenants

- à construire leur identité langagière et culturelle en y intégrant une expérience diversifiée de l'altérité;

- à développer leurs capacités d'apprenants à travers cette même expérience diversifiée de la relation à plusieurs langues et cultures autres." (Coste, Moore, Zarate 2009 p9/10/12, Version revisitée de Compétence plurilingue et pluriculturelle, Conseil de l'Europe, www.coe.int/t/dg4/.../Competence Plurilingue)

La prise en compte des langues de l'environnement des enfants a déjà permis de réaliser la diversité et la multiplicité des situations linguistiques et sociales de chaque établissement, et par là, de participer de la construction d'une image de l'Europe en tant que territoire porteur d'une richesse culturelle intra et inter-continentale. L'intérêt sera de transmettre de façon la plus pédagogique possible cette réalité à l'ensemble des élèves impliqués dans l'opération "sacs d'histoires".

Présenté dans plusieurs séminaires ou colloques en France, Italie et Espagne, c'est le caractère de transférabilité de notre projet qui 
Un projet pour

l'éducation plurilingue

et interculturelle

M.-A. JULLIAN

\section{CONCLUSION}

ÉLÉMENTS DE BIBLIOGRAPHIE: suscite l'intérêt majeur d'autres institutions d'éducation et de formation, ce qui n'est pas sans intérêt pour un développement des objectifs didactiques d'enseignement des langues auquel il a été fait référence, et par la prise en compte indispensable des particularités linguistiques locales ou régionales (5). Si l'on veut consolider une valeur fondamentale de cohésion à l'entité Europe, cette démarche me semble porter sa modeste contribution.

Si j'ai tenté de montrer ou démontrer en quelques lignes que notre projet est porteur d'ouverture sur le plurilinguisme et l'interculturalité, la présence dans cette publication est pour moi une preuve de son intérêt dans la construction en devenir d'une société plurilingue, quelle que soit la langue porteuse d'initiatives, quand bien même ce serait une ou des langues régionales. Pour cette prise en considération non encore établie à tous les niveaux, je tiens à en remercier les responsables. Je souhaite reprendre un passage extrait d'un article paru dans Éducation et Société plurilingues:

"Ce que constate J. Cummins au Canada est valable aussi pour la France et d'autres pays européens: «Le capital culturel, linguistique et intellectuel de nos sociétés augmentera de manière significative quand nous aurons fini de considérer les enfants différents, sur le plan linguistique et culturel, comme un "problème à résoudre" et quand nous nous ouvrirons aux ressources linguistiques, culturelles et intellectuelles que ces enfants apportent à nos écoles et à nos sociétés.» (Abdelilah Bauer, 2010)

Le plurilinguisme et l'interculturalité restent bien un grand projet.

Abdelilah Bauer B. 2010. Etre bilingue en France aujourd'hui, ESP n²9 (décembre): 75-84.

Cavalli M., Coste D., Crisan A. van de Ven P-H. 2009. L'éducation plurilingue et interculturelle comme projet, Division des Politiques linguistiques, Conseil de l'Europe, Strasbourg.

Coste D., D. Moore et G. Zarate. 2009. Compétence plurilingue et pluriculturelle, version révisée de la parution initiale de 1997, Conseil de l'Europe, Strasbourg.

Cavalli M. 2005. Éducation bilingue et plurilinguisme, le cas du Val d'Aoste, LAL Didier Paris

Candelier M. 2008. Approches plurielles, didactique du plurilinguisme: le même et l'autre. Les cahiers de l'Acedle, volume 
Un projet pour

l'éducation plurilingue

et interculturelle

M.-A. JULLIAN
$5, \mathrm{n}^{\circ} 1$ Recherche en didactique des langues - L'Alsace au cœur du plurilinguisme

Duverger J. 2011. Les pédagogies de projets bilingues: des pratiques à privilégier dans les dispositifs bilingues, $\mathrm{ADEB}$ Enseignement bilingue, Le professeur de "Disciplines Non Linguistiques", Paris.

\section{NOTES}

(1) Tous les auteurs ne sont pas cités ici, certains sont dans la bibliographie.

(2) Liste des13 titres: La péniche qui veut des couleurs - La cornemuse languedocienne - La toupie égarée - La petite souris qui cherchait un mari - Pourquoi les grenouilles ont une longue langue Le dragon de Loo - Morena - Cornetta - La légende de l'édelweiss - Les trois jours de la merlette Gune - Le glacier du Ruitor - Monella.

(3) Les membres en étaient: Rita Decime (Inspectrice honoraire, Vice présidente du CIEBP Aoste), Gabriella Vernetto (Inspectrice Assessorat Aoste), Christiane Dunoyer (Ethnologue, Gentre d'études francoprovençales Aoste), Luisa Revelli (Professeur Université Aoste), Claire Torreilles (IPR honoraire en Langue et culture occitanes Montpellier), Élisabeth Zurbriggen (Département éducation Genève), Jean Duverger (Président de l'ADEB, professeur formateur honoraire à l'ISEN), Gérard Roques (IEN Narbonne France)

(4) Un seul collège a participé au projet et a contribué à l'histoire interculturelle et plurilingue de Monella, conte imaginé par 3 classes de niveau Cycle 3 et collège.

Cf. Duverger J. - Précautions pour un enseignement bilingue efficace: "...Complémentairement, une quatrième condition est d'approcher très vite, sous des formes plus légères, d'autres langues prises dans le capital de l'école, de l'environnement proche ou plus lointain: utiliser les langues régionales si elles existent, les langues des élèves issus de l'immigration, les langues du voisin, etc." article paru dans Le français dans le Monde. 